\title{
PKS 0537-441: Extended [O II] emission and a binary QSO?`
}

\author{
J. Heidt ${ }^{1}$, K. Jäger ${ }^{2}$, K. Nilsson ${ }^{3}$, U. Hopp ${ }^{4}$, J. W. Fried ${ }^{5}$, and E. Sutorius ${ }^{6}$ \\ ${ }^{1}$ Landessternwarte Heidelberg, Königstuhl, 69117 Heidelberg, Germany \\ 2 Universitätssternwarte Göttingen, Geismarlandstr. 11, 37083 Göttingen, Germany \\ 3 Tuorla Observatory, 21500 Piikkiö, Finland \\ ${ }^{4}$ Universitätssternwarte München, Scheinerstr. 1, 81679 München, Germany \\ 5 Max-Planck-Institut für Astronomie, Königstuhl 17, 69117 Heidelberg, Germany \\ ${ }^{6}$ Royal Observatory Edinburgh, Blackford Hill, Edinburgh EH9 3HJ, UK
}

Received 25 March 2003 / Accepted 19 May 2003

\begin{abstract}
We present high-resolution imaging and low-resolution spectroscopy of the BL Lac object PKS 0537-441 $(z=0.893)$ and its environment carried out with the ESO-NTT and VLT telescopes. The observations were designed to clarify, whether the properties of PKS 0537-441 are affected by gravitational microlensing due to the claimed detection of a galaxy along the line-of-sight to the BL Lac, or whether PKS 0537-441 and its environment act as a lensing system itself, as suggested by the detection of several closeby companion galaxies with similar morphologies close to PKS 0537-441.

Our observations show that neither case seems to be likely. Within our images we did not find a galaxy along the line-of-sight to the BL Lac as claimed previously. In addition, our spectroscopy shows that none of the four closest companion galaxies (including one new detection by us) is at high redshift. Instead, two of the four nearby companion galaxies to PKS 0537-441 are within $200 \mathrm{~km} \mathrm{~s}^{-1}$ of the systemic velocity of the BL Lac ( $z=0.892$ and 0.895 , respectively). The third companion galaxy is at higher redshift $(z=0.947)$. The fourth companion galaxy shows evidence of $\mathrm{Mg}$ II absorption redwards of its systemic velocity and is perhaps a mini low-ionization BAL QSO at $z=0.885$. If the latter can be confirmed, PKS 0537-441 is the first BL Lacertae object being a member of a binary Quasar.

While we do not find (micro)lensing effects important for this source or its environment, our observations revealed a highly interesting feature. We detected extended [O II] emission in the off-nuclear spectrum of PKS 0537-441, which is most likely due to photoionization from the active nucleus, although we can not rule out the possibility that the extended emission is due to jet-cloud interaction with the counterjet of PKS 0537-441.

According to our analysis of the photometric data, PKS 0537-441 seems to be located in a cluster environment as rich as Abell type $0-1$. This is supported by the detection of four galaxies in the field with similar redshifts as the BL Lac $(\Delta z<0.002)$. However, we serendipitously found even more galaxies at somewhat higher redshifts $(z=0.9-1)$. Thus, PKS 0537-441 might be located in front of a galaxy cluster at somewhat higher redshift or even be part of a large-scale structure with an extension towards the BL Lac.
\end{abstract}

Key words. methods: data analysis - galaxies: distances and redshift - galaxies: active galaxies: BL Lacertae objects: individual: PKS 0537-441 - quasars: emission lines

\section{Introduction}

BL Lac objects are a relatively rare and extreme subclass of the Active Galactic Nuclei (AGN). They are dominated by non-thermal emission from radio- up to $\mathrm{X}$-ray frequencies (in some cases even up to the $\gamma$-ray regime) with mostly weak or even absent emission lines in their optical spectra. Within the

Send offprint requests to: J. Heidt,

e-mail: jheidt@lsw.uni-heidelberg.de

$\star$ Based on observations collected with the VLT-UT1 on Cerro Paranal (Chile) and the NTT on La Silla (Chile) operated by the European Southern Observatory in the course of the observing proposals 64.P-0230 and 66.B-0125. standard AGN picture, their non-thermal radiation emerges from a beamed, relativistic jet closely aligned along the lineof-sight to the observer (Urry \& Padovani 1995).

One of their defining characteristics is their rapid and strong variability across the whole electromagnetic spectrum. There is common agreement that the observed variability is intrinsic to the sources (see e.g. the review by Wagner \& Witzel 1995), although some BL Lac objects may indeed be microlensed background QSOs (Ostriker \& Vietri 1985).

According to the Unified Scheme for AGN, the parent population of BL Lac objects - i.e. those sources, whose jets are misaligned to the observer - are the FR I radio galaxies (Urry \& Padovani 1995). This can be tested by comparing their isotropic 
(unbeamed) properties (e.g. host galaxies or cluster environment), which should be very similar. Host galaxy studies allow us to test the micro-lensing scenario, since here an offset between the center of a detected galaxy and the AGN itself could be present. In this case the galaxy is presumably a system along the line of sight rather than the host of the AGN.

In recent years, in more than $100 \mathrm{BL}$ Lac objects with $z<0.5$ a host galaxy has been detected (e.g. Falomo 1996; Wurtz et al. 1996; Heidt et al. 1999; Falomo \& Kotilainen 1999; Scarpa et al. 2000; Nilsson et al. 2003) and there is common agreement that BL Lac host galaxies are luminous, giant elliptical galaxies. Host galaxy data for BL Lacs at higher redshifts are scarce, so any evolutionary trends can not be investigated. The cluster environment has been studied by Wurtz et al. (1997) for a sample of $\sim 50$ sources up to $z=0.65$ (mostly at $z<0.4$ ). They found an apparent evolution towards denser cluster environments with redshift. However, the apparent evolution is tied to observations of a few BL Lac objects between $z=0.5$ and 0.65 in their sample only. Similar conclusions have been drawn by Fried et al. (1993) from a study of the 1 Jy sample of BL Lacs (Stickel et al. 1993). Unfortunately, due to the limited depth of their observations, they were not able to evaluate the cluster environment for high-redshift $(z=0.8-1) 1 \mathrm{Jy}$ BL Lacs (including PKS 0537-441).

Up to now, there is only one clear example of a lensed BL Lac (B2 0218+35.7, Patnaik et al. 1993). Other objects, whose properties may be affected by gravitational (micro)lensing are AO 0235+164, PKS 0537-441 and B2 1308+326 (see Heidt 1999 for details). These three BL Lacs all have similar properties. They are at relatively high redshift $(z \sim 1)$, are among the most luminous BL Lac objects $\left(M_{R}<-27.5\right)$ and have historically shown the largest variability amplitudes $(\Delta m>5 \mathrm{mag}$, see Table 1 in Stickel et al. 1993). A few more candidates detected during the course of the BL Lac HST snap survey are discussed in Scarpa et al. (1999).

Of particular interest is the BL Lac object PKS 0537-441 $(z=0.892$, Lewis \& Ibata 2000), which is a member of the $1 \mathrm{Jy}$ sample of BL Lac objects (Stickel et al. 1993). It displayed intraday variability at radio (Romero et al. 1995) and optical frequencies (Heidt \& Wagner 1996; Romero et al. 2000), and is one of the few BL Lac objects, where $\gamma$-ray emission has been detected (Hartman et al. 1999). Its continuum variations throughout the entire electromagnetic spectrum have been discussed by Pian et al. (2002). Interestingly, Romero et al. (1995) derived from their radio variability measurements brightness temperatures in excess of $10^{21} \mathrm{~K}$, which is 9 orders of magnitude above the inverse Compton limit. Based on their claimed detection of a galaxy along the line-of-sight to PKS 0537-441 Stickel et al. (1988, see below) discussed, whether the BL Lac could be microlensed by small masses in this foreground galaxy. On the other hand, spectral-index variations found from optical two-color photometry by Romero et al. (2000) seem to rule out the microlensing hypothesis at least for these observations.

PKS 0537-441 was also subject to several broad-band imaging studies through a R-filter, however, with puzzling results. Stickel et al. (1988) claimed the detection of a foreground disk galaxy to PKS 0537-441, with a morphology and brightness similar to a galaxy $11^{\prime \prime}$ east of the BL Lac at $z=0.186$. They concluded that the observed properties of PKS 0537-441 are influenced by gravitational lensing by the foreground galaxy. In subsequent studies Falomo et al. (1992) and Kotilainen et al. (1998) did not detect the foreground galaxy reported by Stickel et al. (1988) and found PKS 0537-441 completely unresolved thus weakening the lensing hypothesis. The former noted two faint companion galaxies close to the BL Lac at $\sim 4^{\prime \prime}$ distance. The BL Lac object also appeared unresolved in HST observations (Scarpa et al. 2000). In a reanalysis of the same HST-image mentioned above, Lewis \& Ibata (2000) found indications for a galactic component after the subtraction of a scaled PSF (which was recently shown to be due to scattered light by Pian et al. 2002). Additionally, they detected 3 companion galaxies close to PKS 0537-441 (2 of which had already been found by Falomo et al. 1992) and discussed an alternative scenario in which PKS 0537-441 is not subject to lensing effects, but is rather a member of a group of galaxies which lense a distant background galaxy. As a consequence at least two of the three companion galaxies could be magnified and split images of a distant background source at high redshift.

Obviously, the results and the interpretation of the results for PKS 0537-441 differ grossly. One major drawback of these studies is that the images were either taken under moderate seeing conditions or suffer from relatively short exposure times. Except for PKS 0537-441 itself and the nearby galaxy $11^{\prime \prime}$ to the east, no spectroscopy in the field of PKS 0537-441 has been carried out until now. In order to clarify the situation, we have undertaken a very deep imaging and multi-object spectroscopic study of PKS 0537-441 and its environment using the NTT and VLT telescopes at ESO, the results of which are presented here. While we will show that lensing effects are most likely not important in this source, several highly interesting features are observed. Amongst them are the presence of extended [O II] emission on the opposite side of the radio jet in PKS 0537-441 and the possible detection of a binary QSO.

To facilitate comparison with other studies, $H_{0}=$ $50 \mathrm{~km} \mathrm{~s}^{-1} \mathrm{Mpc}^{-1}$ and $q_{0}=0$ is assumed.

\section{Observations and data reduction}

\subsection{Imaging}

A very deep I-band image of PKS 0537-441 was acquired at the NTT in service mode on the night November 7/8 1999. The use of the $I$-filter instead of a R-filter (which was used in previous studies) has the advantage that it samples the spectrum redwards of the $4000 \AA$ break at $z=0.893$ thus increasing the chance to detect the host galaxy of PKS 0537-441. We used SUSI2, which consists of a mosaic of two $2 \mathrm{k} \times 4 \mathrm{k}$ EEV-CCDs. The frames were binned by a factor of two, which gives a scale of 0 ' $162 /$ pixel. Thus the field of view is $\sim 5.5 \times 5$ '.5. During the photometric night 43 images $(3 \times 1 \mathrm{~min}, 40 \times 3 \mathrm{~min})$ resulting in an integration time of $123 \mathrm{~min}$ in total were taken. PKS 0537-441 was placed close to the center of one of the two CCDs (CCD \#45, which covers the eastern part of the mosaic). The short individual images were necessary to avoid saturation 

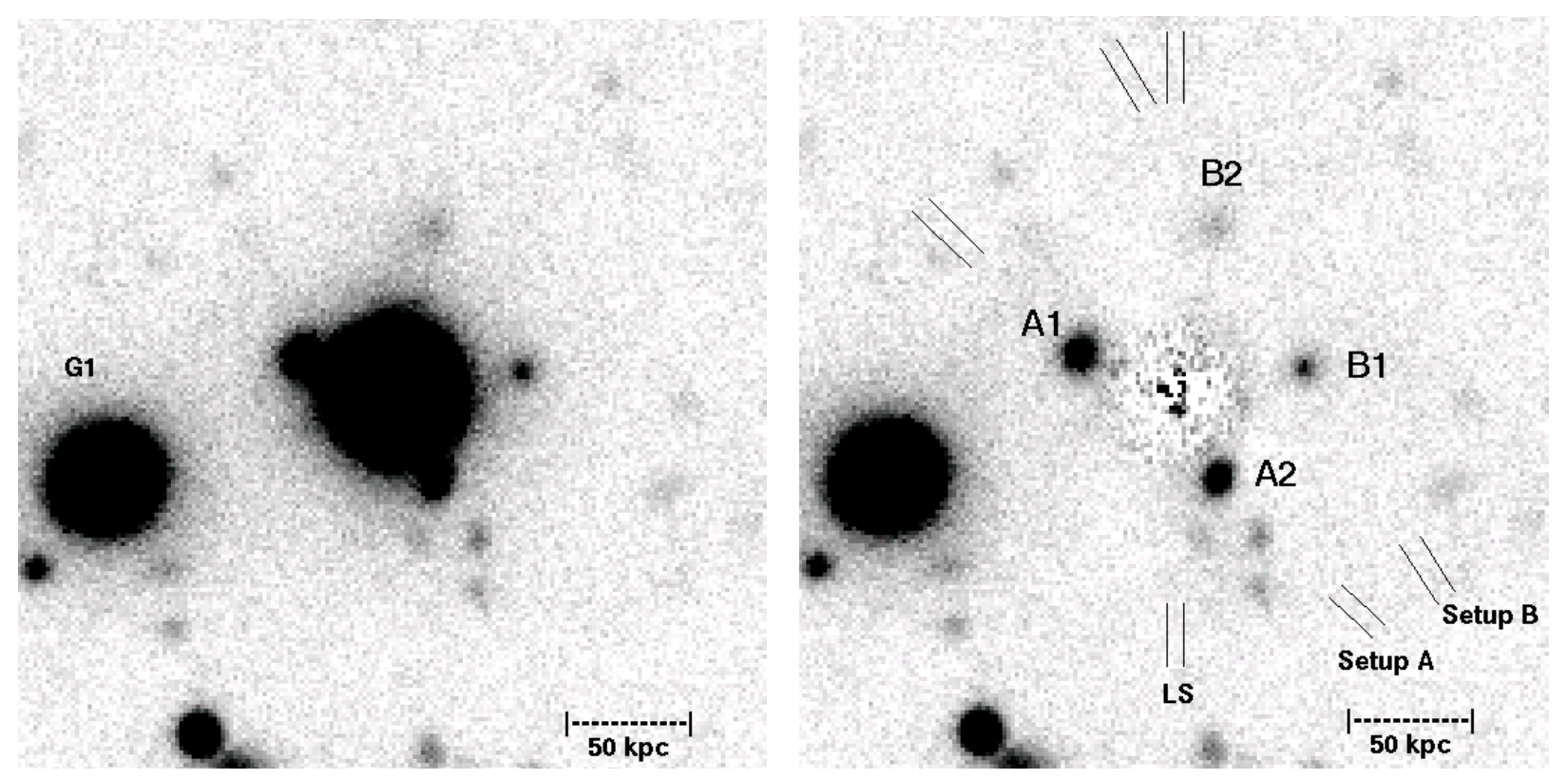

Fig. 1. Left: NTT+SUSI2 I-band image of PKS 0537-441. FOV is $30^{\prime \prime} \times 30^{\prime \prime}$. North is up, east to the left, FWHM 0' 85 . The galaxy G1 11" to the east of PKS 0537-441 at $z=0.186$ is discussed in Stickel et al. (1988). Right: Same image after subtraction of a scaled PSF. The four closest companion galaxies to PKS 0537-441 are labeled. Labeling for A1, A2 and B1 follows Falomo et al. (1992) and Lewis \& Ibata (2000). $\mathrm{B} 2$ is a new detection by us. The orientation of the MOS setups A and B as well as of the longslit spectrum is also indicated.

of the BL Lac nucleus. In order to use the frames for fringe correction and superflat purposes, jittered images were taken. We used a random walk jitter pattern within a rectangular box of $30^{\prime \prime}$ border length centered on the central position. During the night standard stars from Landolt (1992) were observed to set the zero point.

The individual images were first corrected for bias and then for pixel-to-pixel variations. For the latter we used twilight flatfields. A superflat was created from the 43 bias-subtracted and flatfielded images to correct for large-scale gradients. To remove the fringes a scaled fringe template derived from $I$-band images taken during this night was subtracted. Finally, the images were cleaned of cosmic ray hits, aligned and summed. The resulting image has a FWHM of 0. ' 85 .

Additionally, a short $R$-band image of PKS 0537-441 has been taken with the VLT-UT1 and FORS on the night October, 10-11 1999 for the preparation of the spectroscopic observations. The data reduction was similar as for the $I$-band images.

\subsection{Spectroscopy}

Low-resolution spectra of $\sim 46$ objects in the field of PKS 0537-441 were collected with FORS1 at the VLT1 (Antu) during two clear nights on November 19-21, 2000. Observations were done in MOS (Multi-Object Spectroscopy) mode, in which FORS1 provides 19 slitlets of $~ 22^{\prime \prime}$ length in a vertical array. Two MOS-setups were designed. Setup A was observed for 1 hour $(3 \times 20 \mathrm{~min})$ at $\mathrm{PA}=49.6$ (counted counterclockwise from $\mathrm{N}$ ), whereas setup B was observed for 2 hours $(3 \times 40 \mathrm{~min})$ at $\mathrm{PA}=32.6$. We used the grism 150I, which gave us a spectral scale of $\sim 5.5 \AA /$ pixel. The slit width was set to $1^{\prime \prime}$. The wavelength range covered was $\sim 4000-10000 \AA$. Slitlet \#9 for setup A covered two brighter nearby companion objects of PKS 0537-441 (labeled A1 and A2 in Fig. 1), whereas the slitlet \#9 for setup B covered two fainter nearby companion galaxies of PKS 0537-441 (labeled B1 and B2 in Fig. 1). The remaining slitlets were filled serendipitously with galaxies in the field in order to search for galaxies at the redshift of PKS 0537-441. In some cases we were able to put two objects onto one slitlet. The mean DIMM seeing was 0.'6 FWHM for setup A and 1.'05 FWHM for setup B. Finally, a short (10 min) long-slit spectrum across the nucleus of PKS 0537-441 at PA $=0$ with the same slit width and grism was observed on the night November, 21/22 during a period of poor seeing conditions (1." $8 F W H M)$. At the end of each of the nights, spectrophotometric standards from Oke (1990) were observed.

The data reduction of the individual spectra (bias subtraction, flatfielding, cosmic ray removal, sky subtraction, wavelength calibration, etc.) was performed using standard MIDAS routines. The individual spectra of each slitlet were averaged and smoothed using a running mean with a width of 3 pixels. The $F W H M$ spectral resolution measured from strong night sky emission lines is $\sim 25 \AA$.

\section{Analysis and results}

\subsection{Imaging}

\subsubsection{The host galaxy and nearby environment}

In order to search for the host galaxy or a galaxy along the lineof-sight to PKS 0537-441 we applied a fully 2-dimensional fitting procedure to the image (see Nilsson et al. 1999 and Heidt et al. 1999 for details). We first extracted a PSF by averaging the three brightest stars in the field of PKS 0537-441. Only stars present on the same CCD as PKS 0537-441 were 


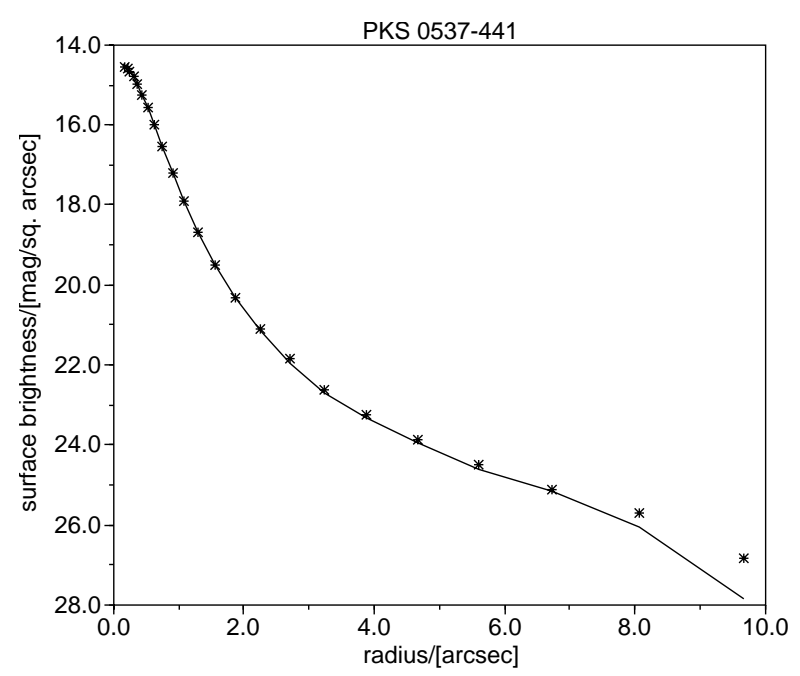

Fig. 2. Surface brightness profile of PKS 0537-441 (asterisks) compared to a scaled PSF (solid line).

used. Unfortunately, PKS 0537-441 was bright during the observations. Thus, even the brightest stars in the field usable for the extraction of the PSF were at least 1.5 mag fainter than the BL Lac itself. In order to improve the $\mathrm{S} / \mathrm{N}$ in the outer parts of the PSF, we smoothed the PSF outside the radius of 2". $4(\sim 3 F W H M)$ using a Gaussian kernel. In the inner part $(r<2$ ". 4$)$ no smoothing was applied in order to accurately preserve the rapidly changing brightness profile close to the center of the PSF. This composite PSF was then used for the model fits.

We fitted three different models to the BL Lac. One representing the AGN (scaled PSF), one representing an AGN + bulge (scaled PSF + convolved de Vaucouleurs model) and one representing an AGN + disk (scaled PSF + convolved disk model). We did not allow for any offset between the AGN and model galaxy, the position angle and ellipticity of the putative host galaxy were set to zero. Prior to fitting, we masked carefully all regions affected by nearby companion objects.

Already the fit with a scaled PSF was an adequate description of the observed light distribution of PKS 0537-441, i.e. we could neither detect the host galaxy of PKS 0537-441 nor a potential galaxy along the line of sight to the BL Lac. PKS 0537-441 appears completely unresolved. Its brightness was $m_{I}=14.45$, which is relatively bright for this source. The image of PKS 0537-441 and its residuals after subtraction of the best fit AGN is shown in Fig. 1, the 1-dim profiles of PKS 0537-441 and a scaled PSF in Fig. 2.

As can be seen from Fig. 1, there are residuals in the center of PKS 0537-441, which are typical for PSFsubtraction. Remarkably, our PSF traces the surface brightness of PKS 0537-441 out to a radius of about 8" covering $\sim 12 \mathrm{mag}$ in brightness (Fig. 2). There are some slight differences at about $2-3^{\prime \prime}$ from the core (seen also as remaining light to the north and west of the BL Lac in Fig. 1), whereas at radii $>8$ " the PSF clearly underestimates the flux from PKS 0537-441. Both features are due to the imperfect PSF.

We have estimated upper limits for the host galaxy brightness using simulated images of PKS 0537-441. The simulated images consisted of a core component with $m_{I}=14.45$ and a host galaxy with effective radius $r_{\text {eff }}=00^{\prime} 95(10 \mathrm{kpc}$ at $z=0.893$ ). Using progressively fainter host galaxies we determined the highest host magnitude that allowed us to make a host detection. For the host galaxy to be detected we required $r_{\text {eff }}^{a v}>5 \sigma_{\text {reff }}$, where $r_{\text {eff }}^{a v}$ is the average effective radius and $\sigma_{\text {reff }}$ is the rms scatter in a set of 30 simulations. Using this method we estimate that a host galaxy with $m_{I}<19.0$ would have been detected.

Inspection of Fig. 1 reveals four resolved galaxies within 7" of PKS 0537-441. The three brightest ones (A1, A2 and B1) have already been detected by Falomo et al. (1992) and Lewis \& Ibata (2000). In addition, there is a fourth companion galaxy $\sim 6$ "' 5 to the north of PKS 0537-441 (labeled B2 in Fig. 1), which is also barely visible in Fig. 1 of Falomo et al. (1992). The brightness of these objects is $m_{I} \sim 21.0,21.2,22.0$ and 23.0 and their projected distances from the BL Lac are 3." 9 , 3". $8,5^{\prime \prime}$ and 6 "' 4 for A1, A2, B1 and B2, respectively.

\subsubsection{Cluster environment}

In order to analyze the cluster environment of PKS 0537-441 we first created an object catalog with positions, magnitudes (MAG_BEST) and a star/galaxy classification index (CLASS_STAR) using SExtractor (Bertin \& Arnouts 1996). MAG_BEST is an isophotal magnitude which considers deblending of objects and applies an aperture flux correction at the edges of sources. The CLASS_STAR parameter can be in the range between 0 (galaxy) and 1 (point source). This value is calculated by a comparison of the $F W H M$ of an image and the morphology of objects. It considers both the ellipticity of objects as well as their ratio of maximum brightness to their extension.

Within the field of $2(\mathrm{CCDs}) \times 2 ! 32 \times 5$ ' 15 we detected $\sim 1200$ sources. After a careful visual inspection of the detections, $\sim 60$ obviously wrong entries were removed (false detections close to the edges of the frame or imperfectly removed cosmic ray hits) and a few sources were added (which were not detected by SExtractor but clearly present). In the latter case we performed aperture photometry with standard Midas routines. The completeness limit derived from the peak in the differential galaxy number counts is $m_{I} \sim 24.5$. Finally, the projected distance (in kpc) of each source to the BL Lac was calculated.

To determine the strength of galaxy clustering around PKS 0537-441 we measured the surface galaxy density as a function of projected distance from the BL Lac. All sources within five circular annuli with equidistant radii from $100 \mathrm{kpc}$ to $500 \mathrm{kpc}$ centered on PKS 0537-441 were counted and their resulting counts compared to those derived for field sources. Precisely, we calculated

$\omega(\theta+\Delta \theta)=\frac{N_{\mathrm{B}}(\theta+\Delta \theta)}{N_{\mathrm{F}}(\theta+\Delta \theta)}-1$

where $N_{\mathrm{B}}(\theta+\Delta \theta)$ are the number counts within a given area $\theta+\Delta \theta$ from the BL Lac and $N_{\mathrm{F}}(\theta+\Delta \theta)$ are the counts which would be expected if the galaxies are distributed as in the field. The mean field galaxy density $N / \mathrm{arcmin}^{2}$ was calculated from galaxy counts at $\theta>500 \mathrm{kpc}$ from which $N_{\mathrm{F}}(\theta+\Delta \theta)$ has been 

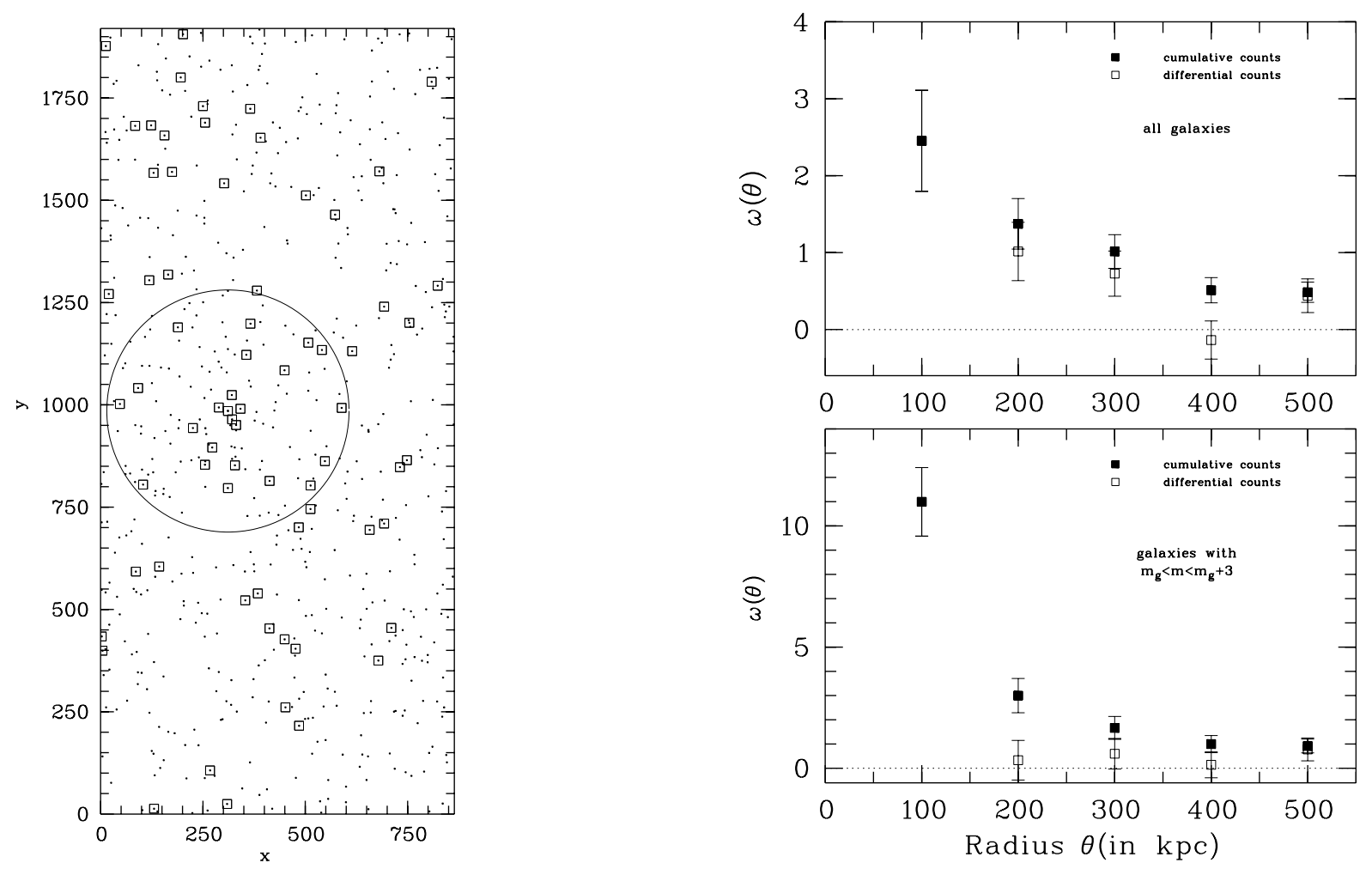

Fig. 3. Left: The field around PKS 0537-441 from the NTT $I$-band image. The $500 \mathrm{kpc}$ environment is indicated by a circle centered on PKS 0537-441. Plotted are all objects with CLASS_STAR $<0.5$ (i.e. galaxies). Galaxies in the magnitude range $m_{\mathrm{g}}<m<m_{\mathrm{g}}+3$ are marked by a square. PKS 0537-441 is included as a galaxy, according to the magnitude-redshift relation by Eales $(1985)$ we used $m_{\mathrm{g}}(I)=19.5$. A density excess towards the BL Lac can easily be seen. There are 24 galaxies within and 47 galaxies outside the $500 \mathrm{kpc}$ region, i.e. $1 / 3$ of the galaxies are within $1 / 6$ of the area covered by this CCD. Right: The galaxy density excess $\omega(\theta)$ as a function of radial distance to the BL Lac. The mean field galaxy density is indicated by the horizontal line. It has been derived from the entire field (i.e. both $2 \mathrm{k} \times 4 \mathrm{k}$ CCDs) excluding the $500 \mathrm{kpc}$ environment of the BL Lac. The top panel shows $\omega(\theta)$ for all galaxies, whereas in the bottom panel only galaxies within the range $m_{\mathrm{g}}<m<m_{\mathrm{g}}+3$ have been used. For both diagrams only galaxies (CLASS_STAR $<0.5$ ) are considered.

scaled according to the area covered. Both, cumulative counts as well as differential counts have been measured.

Figure 3 (top right) shows $\omega(\theta+\Delta \theta)$ by using all galaxies detected (CLASS_STAR $<0.5)$. Even without any restriction of the galaxies to a certain magnitude range a density excess towards PKS 0537-441 can be seen. The clustering signal becomes even stronger if we use only galaxies in a magnitude range, which has the highest probability to be at the BL Lac redshift (Fig. 3 bottom right). For the definition of the magnitude range we followed Hill and Lilly (1991, hereafter HL91) and considered only galaxies within $m_{\mathrm{g}}<m<m_{\mathrm{g}}+3$. The typical apparent magnitude $m_{\mathrm{g}}$ of a giant elliptical galaxy at a given redshift was obtained from the magnitude-redshift relation for radio galaxies of Eales (1985)

$m_{\mathrm{g}}(R)=21.05+5.3 \log z$.

Since we observed in the $I$-band, $m_{\mathrm{g}}(I)$ was estimated by subtracting the $R-I$ color of an early type galaxy (k-correction and galactic extinction towards PKS 0537-441 had also been taken into account).

For an estimate of the cluster richness and a direct comparison to other studies, we calculated the excess of galaxy counts $N_{0.5}$ as defined by HL91. This is the number of galaxies ranging from $m_{\mathrm{g}}<m<m_{\mathrm{g}}+3$ within the $0.5 \mathrm{Mpc}$ radius around the $\mathrm{BL}$ Lac minus the average number counts of the field galaxy distribution. For PKS 0537-441 we find $N_{0.5}=11.5 \pm 3.5$. This corresponds to a spatial cross-correlation amplitude of the order of $B_{q g} \approx 435$ following the relation $B_{q g}=(37.8 \pm 10.9) N_{0.5}$ given by Wold et al. (2000), and hence to a cluster of Abell richness class 0-1 (if compared to the calibration in Table 4 and Fig. 3 of HL91).

\subsection{Spectroscopy}

Preliminary results of the spectroscopic observations have been described by us in Heidt et al. (2001a,b, 2003a). These were solely based on the identification of emission/absorption lines in the spectra. For the present paper more accurate redshifts could be determined by a more sophisticated analysis, which further allowed us to roughly classify the objects into galaxy types.

The spectra were first corrected for the atmospheric $A$ band and $B$-band using the standard stars during the same nights. This is very important, since the $A$-band coincides at $z \sim 0.9$ (the BL Lacs redshift) with the $4000 \AA$ break in early-type galaxies. Then we searched for obvious emission/absorption line features in our spectra and determined their centroids by fitting Gaussians to them. In order to avoid 
Table 1. Classified objects from the 2 MOS-setups in the field of PKS 0537-441.

\begin{tabular}{|c|c|c|c|c|c|c|c|c|c|}
\hline Slitlet $^{1}$ & $\begin{array}{l}\Delta \alpha \\
{\left[{ }^{\prime \prime}\right]}\end{array}$ & $\begin{array}{l}\Delta \delta \\
{\left[{ }^{\prime \prime}\right]}\end{array}$ & $\begin{array}{c}m_{R} \\
{[\mathrm{mag}]}\end{array}$ & $\begin{array}{c}M_{R} \\
{[\mathrm{mag}]}\end{array}$ & $\begin{array}{c}m_{I} \\
{[\mathrm{mag}]}\end{array}$ & $\begin{array}{c}M_{I} \\
{[\mathrm{mag}]}\end{array}$ & $z$ & SED/AGN & comments \\
\hline$\# \mathrm{a} 2$ & 180 & 22 & 23.7 & -21.9 & 22.4 & -23.1 & 1.263 & 3 & {$[\mathrm{O}$ II] } \\
\hline$\#$ a3 & 158 & 13 & 23.5 & -20.2 & 22.4 & -21.3 & 0.658 & 2 & [O II $]$ \\
\hline$\# \mathrm{a} 4 \mathrm{a}$ & 93 & 65 & 18.7 & -21.6 & 17.9 & -22.3 & 0.166 & 1 & $\mathrm{Ca} \mathrm{K}+\mathrm{H}$, g-band, $\mathrm{Mg}$ b, $\mathrm{Na} \mathrm{D}$ \\
\hline$\# \mathrm{a} 4 \mathrm{~b}$ & 88 & 61 & 23.3 & -20.2 & 22.4 & -21.0 & 0.604 & 3 & {$[\mathrm{O}$ II $]$} \\
\hline \#a6 & 63 & 24 & 22.9 & -17.4 & & & 0.165 & 5 & [O II], [O III] \\
\hline$\#$ \#7 & -66 & -13 & 23.6 & -19.1 & & & 0.443 & 5 & {$[\mathrm{O} \mathrm{II}], \mathrm{H} \beta,[\mathrm{O} \mathrm{III}]$} \\
\hline$\# \mathrm{a} 8$ & -11 & 48 & 22.4 & -19.9 & 21.7 & -20.6 & 0.387 & 4 & [O II], H $\beta,[\mathrm{O}$ III] \\
\hline$\#$ a9a & 4 & 1 & & & 21.0 & -23.5 & 0.885 & QSO & Mg II, BAL QSO?, A1 \\
\hline$\#$ a9b & 0 & 0 & & & 14.5 & -30.0 & 0.893 & BL Lac & Mg II, [O II], PKS 0537-441 \\
\hline$\# \mathrm{a} 9 \mathrm{c}$ & -2 & -3 & & & 21.2 & -23.5 & 0.947 & 2 & $\mathrm{Ca} \mathrm{K}+\mathrm{H}, \mathrm{Mg}$ b, A2 \\
\hline$\#$ a10 & 0 & -31 & 23.4 & -21.1 & 22.2 & -22.3 & 0.892 & 3 & {$[\mathrm{O}$ II $]$} \\
\hline$\#$ \#11 & -39 & -20 & 23.0 & -15.3 & 22.5 & -15.8 & 0.068 & 3 & $\mathrm{H} \beta,[\mathrm{O} \mathrm{III]}, \mathrm{H} \alpha$ \\
\hline \#a12 & -12 & -100 & 23.9 & & 22.1 & & & & M-star \\
\hline$\#$ \#13 & -24 & -117 & 23.3 & -21.4 & 22.1 & -22.6 & 0.952 & 3 & [O II] \\
\hline$\#$ a15a & -146 & -47 & 23.5 & -19.6 & & & 0.529 & 3 & [O II $]$ \\
\hline$\# \mathrm{a} 15 \mathrm{~b}$ & -153 & -53 & 20.6 & -22.6 & & & 0.555 & 3 & [O II], [O III] \\
\hline \#a17 & -136 & -130 & 23.7 & -21.5 & & & 1.115 & 4 & {$[\mathrm{O} \mathrm{II}]$} \\
\hline \#a18 & -165 & -130 & 24.0 & -21.0 & & & 1.058 & 3 & [O II $]$ \\
\hline$\# \mathrm{~b} 2$ & 141 & 97 & 24.3 & -20.6 & 23.0 & -21.8 & 0.998 & 3 & [O II] \\
\hline \#b3a & 132 & 84 & 23.5 & -19.8 & 22.6 & -20.6 & 0.560 & 3 & [O II $]$ \\
\hline$\# \mathrm{~b} 4$ & 19 & 131 & 23.3 & -21.4 & 22.1 & -22.6 & 0.936 & 2 & [O II], Ca K+H \\
\hline \#b5 & 23 & 94 & 23.7 & -20.9 & 22.1 & -22.5 & 0.916 & 2 & [O II], Ca K+H, g-band \\
\hline \#b6 & 66 & 43 & 23.7 & -18.9 & & & 0.424 & 5 & [O II $], \mathrm{H} \beta,[\mathrm{O} \mathrm{III}]$ \\
\hline$\# \mathrm{~b} 7$ & 46 & 26 & 24.3 & -19.2 & 23.2 & -20.2 & 0.604 & 5 & [O II], [O III $]$ \\
\hline \#b8 & 31 & 17 & 24.3 & -19.4 & 23.2 & -20.5 & 0.652 & 5 & [O II], H $\beta,[\mathrm{O} \mathrm{III]}$ \\
\hline$\#$ \#9a & -5 & 1 & & & 23.0 & -21.5 & 0.892 & 5 & [O II $], \mathrm{B} 2$ \\
\hline$\# \mathrm{~b} 9 \mathrm{~b}$ & -1 & 6 & & & 22.0 & -22.5 & 0.895 & 2 & [O II], Ca K+H, g-band, B1 \\
\hline \#b10 & -6 & -15 & 24.5 & -19.3 & 23.7 & -20.0 & 0.672 & 5 & {$[\mathrm{O} \mathrm{II}],[\mathrm{O} \mathrm{III}]$} \\
\hline$\# \mathrm{~b} 12$ & -34 & -46 & 24.9 & -19.8 & 23.5 & -21.1 & 0.927 & 4 & {$[\mathrm{O} \mathrm{II}]$} \\
\hline$\# \mathrm{~b} 13 \mathrm{a}$ & -19 & -84 & 24.3 & -20.4 & 22.8 & -21.9 & 0.949 & 3 & [O II] \\
\hline$\# \mathrm{~b} 14 \mathrm{a}$ & -65 & -85 & 22.1 & -21.2 & 21.3 & -22.0 & 0.564 & 3 & [O II], [O III] \\
\hline$\# \mathrm{~b} 14 \mathrm{~b}$ & -67 & -88 & 23.8 & -20.7 & 23.3 & -21.2 & 0892 & 4 & {$[\mathrm{O} \mathrm{II}]$} \\
\hline \#b16 & -74 & -144 & 23.5 & -21.2 & 22.4 & -22.3 & 0.948 & 1 & $\mathrm{Ca} \mathrm{K}+\mathrm{H}$, g-band \\
\hline$\#$ b17a & -85 & -162 & 23.5 & -21.4 & & & 0.992 & 1 & $\mathrm{Ca} \mathrm{K}+\mathrm{H}$, g-band \\
\hline$\# \mathrm{~b} 17 \mathrm{~b}$ & -86 & -164 & 23.0 & -21.8 & & & 0.989 & 1 & $\mathrm{Ca} \mathrm{K}+\mathrm{H}$, g-band \\
\hline \#b18 & -90 & -173 & 21.0 & & & & & & M-star \\
\hline
\end{tabular}

${ }^{1}$ Integration time was 1 hour for the \#a* objects and 2 hours for the \#b* objects. In some cases, two objects could be placed onto the slitlet. Those objects were labeled $* \mathrm{a}$ and $* \mathrm{~b}$, respectively.

misidentifications due to artefacts (e.g. imperfect removed cosmic ray signatures), all the $20 \mathrm{~min}$ and $40 \mathrm{~min}$ spectra were inspected and compared individually. Finally, we fitted interactively five different spectral energy distributions (SEDs) to the spectra. As starting values we used preliminary redshifts obtained from emission/absorption lines. The five different SEDs used for the fitting procedure were derived from spectroscopic observations of about 500 galaxies in the FORS Deep Field ${ }^{1}$ (S. Noll, in prep.). They have the advantage that they were

\footnotetext{
${ }^{1}$ See Heidt et al. (2003b) for details on the FORS Deep Field.
}

derived with the same spectroscopic setup, instrument and telescope as the observations presented here. The galaxy types covered are: SED1 = E-S0, SED2 $=$ Sa-Sc, SED3 $=$ Sd/Irr, SED4 = starburst, SED5 = extreme starburst. Four of the SED5 sources (\#a6, \#a7, \#b6 and \#b10) may be AGN. They all have the commonly used flux ratios [O III] 5007/[O II] $>1$, [O III] 4959/H $\beta>1$ and [O III] 5007/H $\beta>3$ for a classification as an AGN. Unfortunately, due to the relatively high redshift of the sources, the important diagnostic lines [N II] 6583/H $\alpha$ are either in the extreme red part of the spectrum dominated by night sky emission or outside the spectral range covered. 

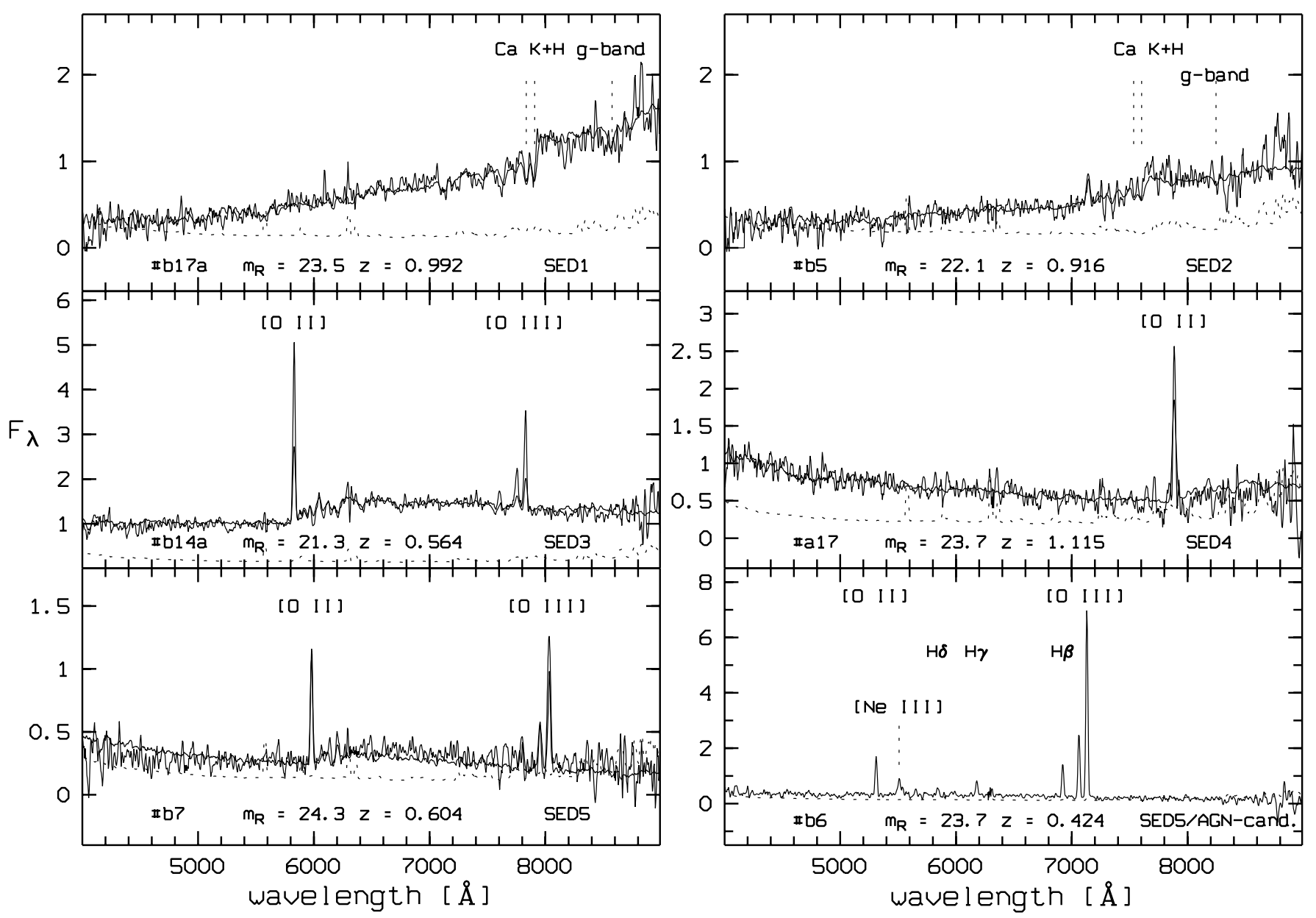

Fig. 4. Spectra of representative galaxies and of an AGN candidate detected in the field of PKS 0537-441. Templates of average spectral energy distributions of galaxies of different Hubble type (SED1-5) are overplotted on the observed galaxies' spectra. The error spectrum (dashed line) is displayed as well.

The ratio [O III] $5007 / \mathrm{H} \beta$ vs. [N II] $6583 / \mathrm{H} \alpha$ is a robust diagnostic diagram to separate starburst galaxies from AGN (Veilleux \& Osterbrock 1987).

The approach of fitting SEDs is especially helpful for the redshift determination for galaxies dominated by an old stellar population. The error in the redshift determination is $\Delta z \sim 0.001$ for sources with at least 2 emission lines and $\Delta z \leq 0.003$ for the remaining sources. The latter error was derived by slightly varying the redshift of the determined SEDs for individual galaxies until an obvious mismatch was apparent.

The results are summarized in Table 1 . Column 1 identifies the slitlet, where the objects were located, Cols. 2 and 3 give the offsets with respect to PKS 0537-441 followed by the apparent and absolute $\mathrm{R}$ and I magnitudes in Cols. 4 to 7 . The apparent $R$ and $I$ magnitudes were measured using SExtractor (Bertin \& Arnouts 1996). Since PKS 0537-441 is strongly saturated on the $R$-band acquisition image taken for the preparation of the MOS setups, we do not give $R$ magnitudes for the companion galaxies $\mathrm{A} 1, \mathrm{~A} 2, \mathrm{~B} 1$ and $\mathrm{B} 2$. The $R$ - and $I$-images do not fully overlap. Hence, $I$ magnitudes could not be derived for all sources. No k-correction was applied to the absolute magnitudes, because the classification into SEDs is very rough. Column 8 gives the redshift and Col. 9 the rough SED classification. Finally, in Col. 10, the detected lines are listed and comments are given. In Fig. 4 representative spectra of objects with SED1-5 and one AGN candidate are shown.

For 36 out of the 46 spectra in total, we were able to determine the nature of the sources. Their $R$ and $I$ magnitudes range from 18 to 24.5 , most have $m_{R}>22.5$. Their absolute magnitudes range from -15.3 to -22.6 (median -20.6 ) in $R$ and from -16 to -30 (median -21.9 ) in $I$. Remarkable is the extreme brightness of the BL Lac (-30.0) and brightness of the two nearby companions A1 (-23.5, QSO) and A2 (-23.5, early-type at $z=0.947)$. We found 32 galaxies (4 SED1, 5 SED2, 12 SED3, 4 SED4 and 7 SED5), 2 AGN (PKS 0537-441 and A1, which will be discussed in the next section) and 2 stars.

For about $60 \%(19 / 34)$ of the galaxies, at least two emission/absorption lines could be detected, which allowed a secure redshift measurement. In 14 sources we could only detect a single narrow emission line, which we identified with [O II] in all cases. This identification was supported in most cases by either a jump of the continuum redwards of the [O II] line 


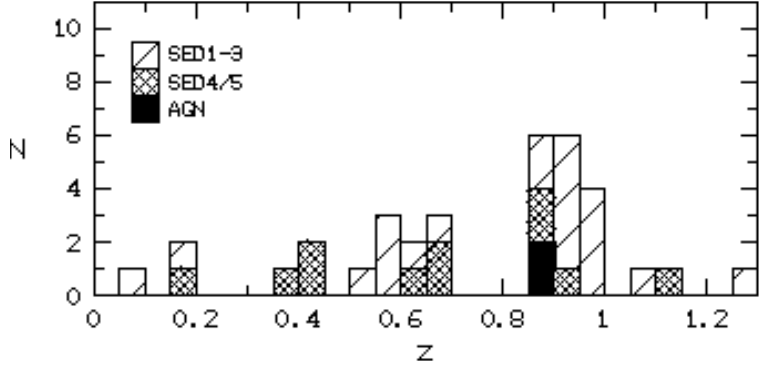

Fig. 5. Distribution of the objects from the 2 MOS-setups with redshift and type. Hatched $=$ SED1-3, double hatched $=$ SED4 $/ 5$ and black $=$ AGN, respectively.

indicating the $4000 \AA$ break or a blue continuum typical for strong star forming galaxies. Finally, in companion galaxy A1, we detected a broad emission line which we identified as Mg II.

The redshifts of the objects range from 0.07 up to $1.3,19$ are at $z>0.8$. Most objects are dominated by [O II] emission, implying relatively high star formation rates. We found also several early-type galaxies at $z>0.9$. Four objects have redshifts similar to PKS 0537-441 $(\Delta z<0.002)$. The distribution of objects with redshift and type is shown in Fig. 5.

\subsubsection{PKS $0537-441$ and $A 1, A 2, B 1 \& B 2$}

In Fig. 6 the 1-dim spectra of PKS 0537-441 and the companion galaxies A1, A2, B1 and B2 are displayed. Although the companion galaxies are about 6-8 mag fainter than the BL Lac, their spectra were sufficiently well separated from PKS 0537-441 to measure not only characteristic lines, but also to detect the continuum. The spectra are discussed below.

PKS 0537-441: In the off-nuclear spectrum of PKS 0537-441, Mg II at $5300 \AA$ and [O II] at $7052 \AA$ are detected, which give $z=0.893$ on average. In the spectrum across the nucleus, only Mg II at $5300 \AA(z=0.894)$, but not [O II] was unambiguously detected. Both lines have previously been found by Lewis \& Ibata (2000) on their nuclear spectra with higher resolution. They give $z=0.892$ in good agreement with our measurement. We also note that [O II] has not been detected by Stickel et al. (1993) and by Falomo et al. (1994), although it may be present in the spectrum shown by Stickel et al. (cf Fig. 2 in their paper).

A1: The blue spectrum of this object shows a very broad asymmetric line at $5275 \AA$ and a narrow line at $7052 \AA$, which are most likely Mg II and [O II]. The Mg II line is $\sim 25 \AA$ blueshifted with respect to the Mg II line in PKS 0537-441 and gives $z=0.885$. It can not be $\operatorname{Ly} \alpha$ at $z=3.338$ since the continuum extends towards the blue and no obvious Ly-break is present. We emphasize here that the broad asymmetric line at $5275 \AA$ can also not be scattered light from the Mg II line in PKS 0537-441 due to reflections inside FORS. This line in A1 can always be seen with a similar structure in all 3 individual images. Moreover, reflections inside FORS normally scale as $10^{-4}$ (W. Seifert, priv. com.), whereas we observe here a difference of the $\mathrm{Mg}$ II lines of the order of $10^{-1}$. However, the center of the [O II] line in A1 is at the same position as the [O II] line in the off-nuclear spectrum of PKS 0537-441.
In order to investigate the apparent discrepancy between the $\mathrm{Mg}$ II and [O II] lines, we subtracted the continuum on the 2-dim spectrum of A1, A2 and PKS 0537-441. This was obtained by fitting and subtracting row-by-row a smoothed cubic spline from the 2-dim spectrum. Great care was taken to match the continuum next to $5300 \AA$ and $7050 \AA$ as well as possible. In Fig. 7 the 2-dim averaged, smoothed spectrum and the resulting continuum-subtracted spectrum are shown. There are several remarkable features. First of all, the $\mathrm{Mg}$ II line of PKS 0537-441 and the Mg II line of A1 are well separated. It is also obvious, that the $\mathrm{Mg}$ II line of A1 is very broad and its center somewhat blueshifted with respect to the $\mathrm{Mg}$ II line in the BL Lac. By contrast, the [O II] line of PKS 0537-441 extends from the central position (at about 1.'7 from the core) with decreasing intensity without any blueshift towards A1. The projected length of that feature is about $4^{\prime \prime}(\sim 40 \mathrm{kpc}$ at $z=0.893$ ). There might also be some (kinematic) substructure (wiggles) present, but this could be due to the continuumsubtraction procedure. Since the central position of $\mathrm{Mg}$ II and $[\mathrm{O} \mathrm{II}]$ in the BL Lac give the same redshift within the errors contrary to the central position of $\mathrm{Mg}$ II in $\mathrm{A} 1$ and since the intensity of the [O II] line decreases towards A1 without any blueshift, we believe that [O II] is intrinsic to PKS 0537-441. Curiously, [O II] does not extend in the opposite direction. A physically plausible explanation for this complex configuration will be discussed in the next section.

A2: The spectrum of this galaxy is dominated by a red continuum showing $\mathrm{Ca} \mathrm{K}+\mathrm{H}$ and $\mathrm{Mg}$ b absorption lines characteristic for early-type galaxies with SED2. No emission lines are detected. The redshift of this galaxy is $z=0.947$.

B1: This galaxy has also a relatively red spectrum (SED2) showing $\mathrm{Ca} \mathrm{K}+\mathrm{H}$ and the g-band in absorption as well as a faint [O II] emission line. The resulting redshift is $z=0.895$, close to the redshift of PKS 0537-441.

B2: Similarly to A1, the spectrum of this source has a blue continuum (SED5) and a relatively strong narrow emission line, which we identify with [O II]. The resulting redshift is $z=0.892$, again, close to the redshift of PKS 0537-441.

\section{Discussion}

Although our I-band image of PKS 0537-441 is probably the deepest ever taken of that field, we were not able to resolve the host galaxy of the BL Lac. This might be at least in part due to the absence of sufficiently bright stars in the field which prevented us to extract a very good PSF. The lower limit to the host galaxy magnitude based on our simulations is $m_{I}=19.0$, which would result in $M_{I}=-26.8$ (k-correction included). With a typical color of ellipticals of $R-I=0.7$ (Fukugita et al. 1995) this would give $M_{R}=-26.1$. Thus, using a typical BL Lac host magnitude of $M_{R}=-23.5$, our observations should have been at least 3 mag deeper given the less than optimal PSF. On the other hand, we can rule out an intervening galaxy as bright as the early-type companion galaxy G1 $\left(m_{I} \sim 17.4\right) 11^{\prime \prime}$ to the east, which was seen by Stickel et al. (1988). This confirms the observations by Falomo et al. (1992), Kotilainen et al. (1998) and Scarpa et al. (2000). Clearly, we can not rule out an even fainter galaxy along the line of sight (our detection limit is 


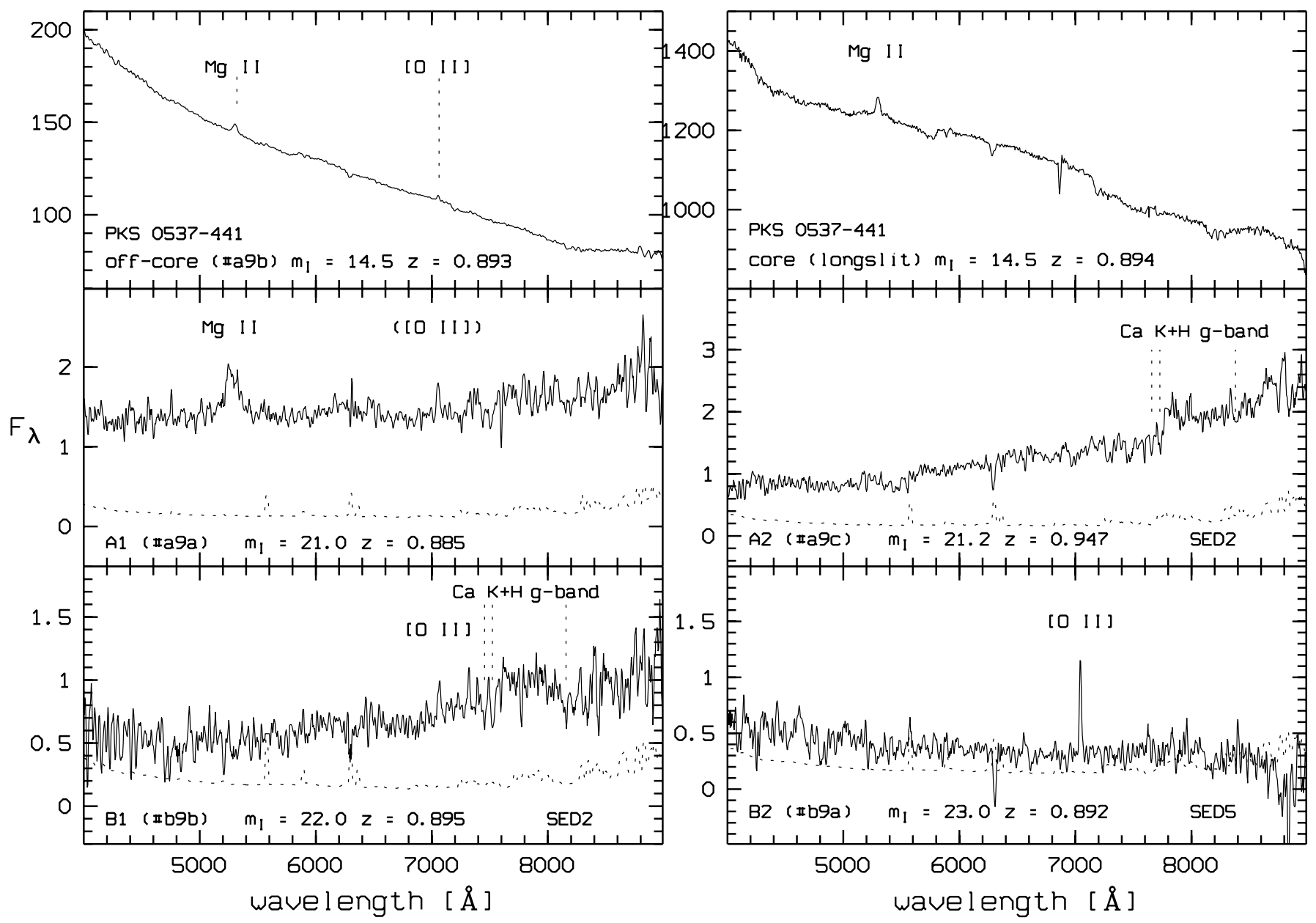

Fig. 6. Spectra of PKS 0537-441 (off-nucleus from setup A and across the core from longslit observations) and of the 4 companion galaxies A1, A2, B1 and B2. For the companion galaxies the error spectrum (dashed line) is included.

about $\mathrm{L}^{*}$ at $\left.z \sim 0.2\right)$. Nevertheless, there is no strong evidence that the properties of this very luminous and strongly variable BL Lac nucleus are affected by gravitational microlensing effects. This is further supported by the absence of absorption lines in HST FOS spectra (Bechtold et al. 2002). A deep high-resolution image of PKS 0537-441 in a very low state is required to unambiguously detect the host galaxy or a faint galaxy along the line of sight, but this is hard to accomplish.

To test, if some of the nearby companion galaxies are lensed background sources, we have carried out multi-object spectroscopy in the field of PKS 0537-441 including the four companion objects. None of the four nearby companion galaxies was found to be at high redshift, i.e. it also does not seem that the environment of PKS 0537-441 acts as a lensing system.

On the other hand, our spectra show that PKS 0537-441 and the companion galaxies A1, A2, B1 \& B2 form a very complex system. Whereas A2 is an early-type galaxy at $z=0.947$, the companion galaxies B1 and B2 $(z=0.895$ and 0.892 , respectively) are within $200 \mathrm{~km} \mathrm{~s}^{-1}$ systemic velocity of the BL Lac, i.e. they are very close to PKS 0537-441. Due to their proximity to PKS 0537-441 they could trigger the strong activity in this source via gravitational interaction.
The relation between PKS 0537-441 and A1 is very hard to interpret. Whereas we detected $\mathrm{Mg}$ II in both, the nuclear and off-nuclear spectrum of PKS 0537-441, [O II] was only detected in the latter. If one compares the relative strengths of $\mathrm{Mg}$ II in the two spectra (cf Fig. 6), one would also expect to see $[\mathrm{O}$ II] in the nuclear spectrum, which is, however, not present. We also failed to detect [O II] in the nuclear 2-dim spectrum by subtracting the continuum in the same way as described for the off-nuclear spectrum. This points to the fact that the [O II] emission is only excited in that region, which is supported by the detection of extended, asymmetric [O II] emission with decreasing intensity towards A1 in the 2-dim offnuclear spectrum.

There are several mechanisms, which may give rise to the $[\mathrm{O}$ II] emission starting $\sim 20 \mathrm{kpc}$ from the core and extending $\sim 40 \mathrm{kpc}$ outwards: locally induced star formation due to gravitational interaction, photoionization by the central active nucleus or shock-ionization via interaction between radio-emitting components and the ambient gas (jet-cloud interaction).

In order to induce star formation by gravitational interaction a companion galaxy or at least a merger remnant is required. We think that this is unlikely here. No companion 

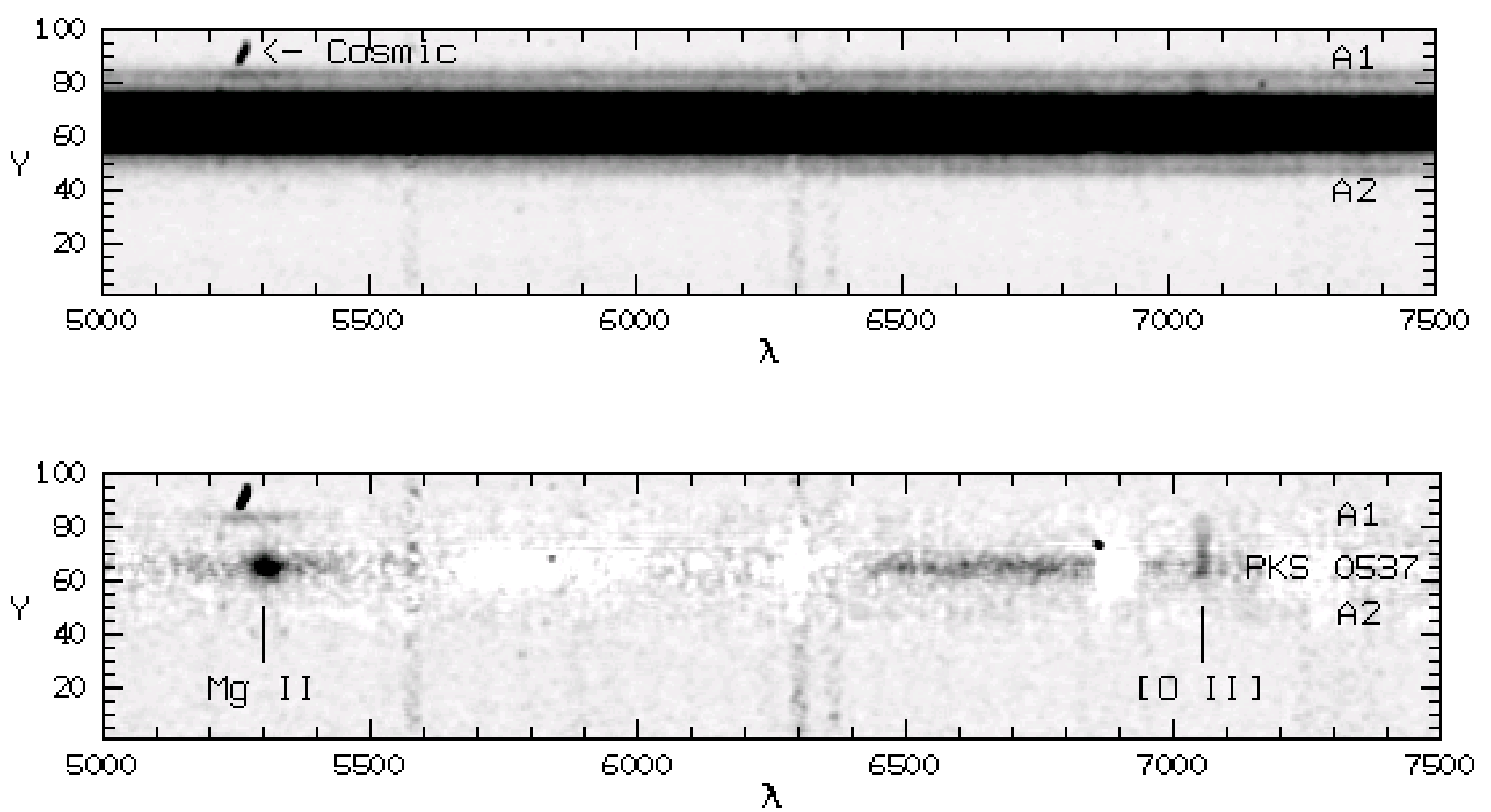

Fig. 7. Top: 2-dim spectrum of slitlet \#9 from setup A. The positions of the companion galaxies A1 and A2 are displayed. A cosmic ray hit is also indicated. The spectra of both companion galaxies are well separated from the off-nuclear component of PKS 0537-441. Note the relatively blue continuum of A1 and the relatively red continuum of A2. The broad Mg II emission at $~ 5275 \AA$ in A1 and some faint extended [O II] emission at $7052 \AA$ from the BL Lac towards A1 can be seen. Bottom: The same spectrum after subtraction of the continuum. Note that the Mg II emission from A1 is well separated from the Mg II emission of PKS 0537-441, whereas the [O II] emission extends from the BL Lac towards A1. Note also that the [O II] emission does not extend towards the opposite direction. See text for details.

galaxy is detected in that region (A1 is at lower redshift) and no obvious tidal features are present at the location of the extended [O II] region. Moreover, elliptical galaxies normally do not possess much gas at $\sim 3 r_{\mathrm{e}}$ (under the assumption that the host galaxy of PKS 0537-441 is a typical host galaxy with $\left.r_{\mathrm{e}}=10 \mathrm{kpc}\right)$.

Whether photoionization by the active nucleus or jet-cloud interaction is the dominant mechanism depends mainly on the size and extent of the radio sources with respect to the emission line regions (see review by Tadhunter 2002). Photoionization is normally the dominant mechanism for those AGN, where the radio source extends well beyond the emission line regions (Tadhunter 2002) although kinematical evidence for jet-cloud interaction has recently been found in exactly such sources (e.g. Solórzano-Iñarrea et al. 2001).

With our limited information available, we are not able to distinguish between or set even constraints on the last two possibilities described above. Whereas photoionization by the active nucleus is certainly the more realistic explanation (extended line-emission up to $20 \mathrm{kpc}$ from the nucleus has been detected e.g. in the BL Lac PKS 0521-36, Boisson et al. 1989), the alternative can not be ruled out. PKS 0537-441 is one of the few BL Lacs classified as a FR II radio source (Rector \& Stocke 2001 and references therein) and shows in radio images a curved jet-like structure leading to the west (Cassaro et al. 1999). Remarkably, our extended [O II] emission is present almost exactly on the opposite side of the jet of PKS 0537-441 extending $\sim 40 \mathrm{kpc}$ in length. This is illustrated in Fig. 8, where we show a comparison of the location of the jet in PKS 0537-441 and the extended [O II] emission. We may see here at least some signature of a counterjet in this BL Lac. Evidence for a counterjet has been detected via kinematic signatures of jet-cloud interaction (line-splitting in [O III]) in 3C 120 (Axon et al. 1989). We note, that some kinematic signatures in the extended [O II] emission in our 2-dim off-nuclear spectrum of PKS 0537-441 may be present, although this may be due to the continuum-subtraction procedure.

Further inspection of Fig. 8 reveals that the jet in PKS 0537-441 apparently turns over close to B1. This may be due to interaction of the jet with $\mathrm{B} 1$, where a pressure gradient deflects the jet considerably. Again, whether this is correct or not can not be tested by our observations but it is not unusual. A similar feature has been observed in the low-redshift BL Lac 3C 371 albeit on smaller spatial scales (Wrobel \& Lind 1990; Nilsson et al. 1997).

The nature of A1 is also unclear. As we have argued in the previous section, the emission line with some absorption towards the red is most likely $\mathrm{Mg}$ II at $z=0.885$. With $M_{I}=-23.5$ we classify A1 therefore as a QSO (note that our $I$-filter samples almost exactly the $B$-band at $z=0.885$ ). Fitting a Gaussian to the absorbed emission line gives a width of the line of $\sim 100 \AA F W H M\left(\sim 5000 \mathrm{~km} \mathrm{~s}^{-1}\right)$. Corrected for redshift this gives a $F W H M$ of $\sim 53 \AA$, which is much larger as typically measured in QSOs ( 35 $\AA$ FWHM, Vanden Berk et al. 2001). If the absorption is due to an absorbing system along the line of sight to A1 one would also expect absorption in the 


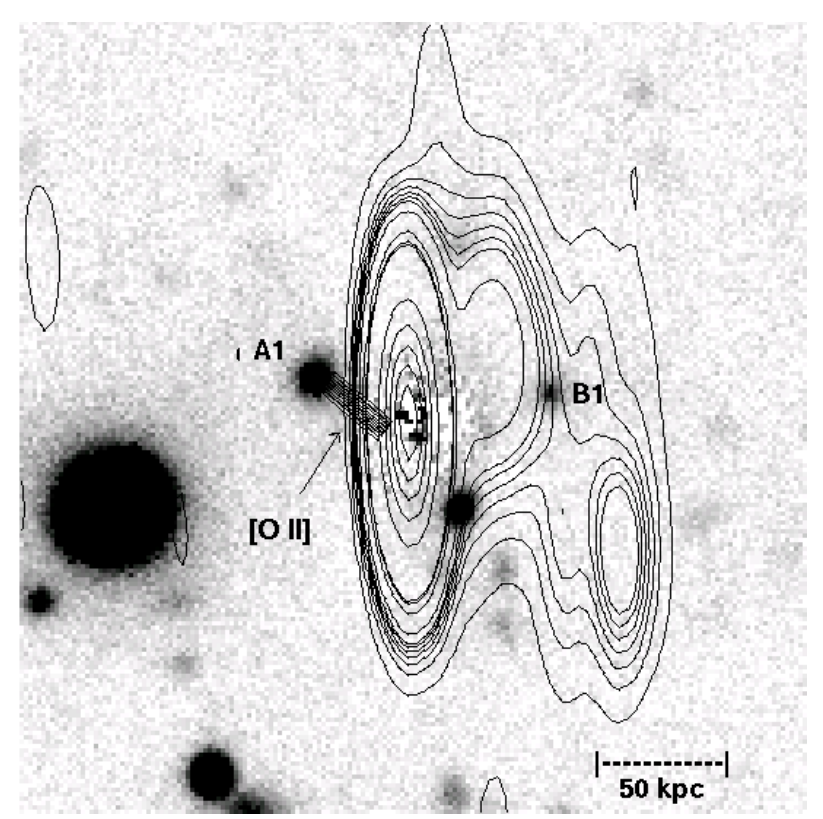

Fig. 8. Same as Fig. 1 with the VLA $1360 \mathrm{MHz}$ image of PKS 0537-441 superimposed (courtesy of P. Cassaro). The region, where the extended off-nuclear [O II] emission towards A1 has been detected is indicated. It is obviously on the opposite side to the jet. Note also the coincidence of the location of $\mathrm{B} 1$ and the turnover of the jet. FOV is $30^{\prime \prime} \times 30^{\prime \prime}$, north is up, east to the left.

spectrum of PKS 0537-441, which is not observed. Therefore, the absorption must be intrinsic to the source and A1 could hence be a mini low-ionization BAL QSO (LoBAL).

Although classical BAL QSOs have their absorption bluewards of their systemic redshifts, a few BAL QSOs with absorption redwards have been detected in the SDSS (Hall et al. 2002). The absorption redwards of the systemic redshift can be either due to infalling gas crossing our line of sight or due to a rotation-dominated disk wind (Hall et al. 2002). Since we do not detect unambiguously [O II] at $\sim 7025 \AA(z=0.885)$ in A1 indicative of a relatively high covering factor, infall of gas could be the dominating absorption mechanism. There is at least indirect support for the identification of $\mathrm{A} 1$ as a mini LoBAL by the non-detection of [O II], which is often absent or weak in Mg II LoBALs (Boroson \& Meyers 1992) or in the compact reddened absorption-line object Hawaii 167 (Cowie et al. 1994). A counterpart to A1 is SDSS 0127+0114 (Hall et al. 2002), which is a strongly reddened mini LoBAL and has a Mg II configuration similar to the one observed in A1. In Hall et al. (2002) there is also a comparison between the dereddened spectrum of SDSS $0127+0114$ and a SDSS composite spectrum (their Fig. 12), which shows the strength of the absorption. We performed a similar comparison by blueshifting the continuum-subtracted BL Lac spectrum by $25 \AA$ and scaling it to match the wings of the absorbed $\mathrm{Mg}$ II emission in A1. The comparison is shown in Fig. 9. As can be seen, the spectra match very well. The resulting $F W H M$ would now be $\sim 40 \AA$ instead of $\sim 100 \AA$.

We investigated the morphology of A1 on our I-band image in order to test for the presence of an AGN. The same analysis as for PKS 0537-441 was performed on the PSF-subtracted

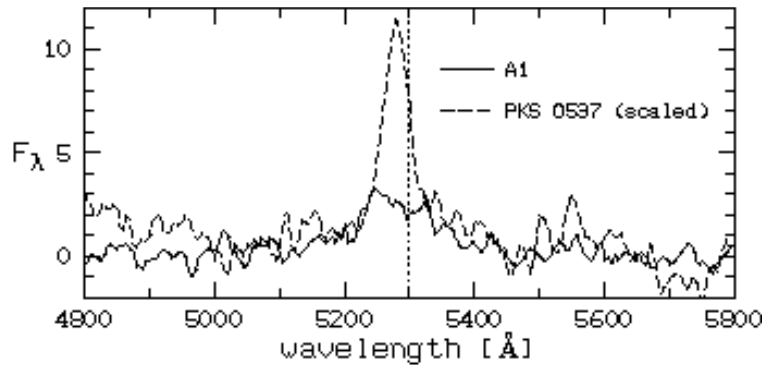

Fig. 9. Comparison of the Mg II lines in A1 (solid line) and the BL Lac (long dashed line). The latter was shifted by $25 \AA$ towards the blue and scaled up by a factor of 2 . The continuum was subtracted in both spectra. Note the good match of the wings of the lines. For comparison the original position of the center of the Mg II line in PKS 0537-441 is indicated.

image (Fig. 1, right). According to our fits, an AGN+galaxy model is not strongly preferred over a pure galaxy model to describe the morphology of A1. Our best fit was an AGN+disk model with $m_{\text {core }}=22.58, m_{\text {disk }}=21.07$ and $r_{\mathrm{e}}=00^{\prime} 61$ $\left(\chi^{2}=1.17\right)$, whereas we got $m_{\text {disk }}=20.9$ and $r_{\mathrm{e}}=0 .{ }^{\prime} 45$ $\left(\chi^{2}=1.36\right)$ for a pure disk model and $m_{\mathrm{deVauc}}=20.59$ and $r_{\mathrm{e}}=0.64\left(\chi^{2}=1.24\right)$ for a pure de Vaucouleurs model, respectively. We could not obtain a good fit with an AGN+de Vaucouleurs model. On the archival HST snapshot image of PKS 0537-441 A1 and A2 are very faint, but A1 appears more compact than A2. Thus there is no direct proof for a compact object in the center of A1, but this may be taken as further evidence for a (reddened) mini LoBAL QSO.

If our favored explanation for the PKS 0537-441 - A1 system is correct, this system would form a Quasar pair. Based on statistical arguments Kochanek et al. (1999) and Mortlock et al. (1999) have shown that the majority of wide separation $\left(3^{\prime \prime}<\Delta \theta<10^{\prime \prime}\right)$ Quasar pairs are rather binary Quasars than gravitational lenses. Our system with a projected separation of 3. .' 9 and with a velocity difference of $\sim 870 \mathrm{~km} \mathrm{~s}^{-1}$ would thus fall into the category of the rare class of binary Quasars. This is supported by the fact that the PKS 0537-441 - A1 system is a $\mathrm{O}^{2} \mathrm{R}$ pair (both components detected in the optical, but only one in the radio; Kochanek et al. 1999), has the most extreme magnitude difference of all QSO pairs and differs strongly in its optical appearance. Given the relative small separation in projected distance and relative velocity (the latter of which may even be smaller), this system may finally merge and form a supermassive binary black hole (Begelman et al. 1980).

We finally remark that the PKS 0537-441 - A1 system would now already be the third binary QSO involving a BAL QSO (including LBQS 0103-2753 (Junkkarinen et al. 2001) and SDSS $0300+0048$ (Hall et al. 2002)). This is a high fraction of BAL QSOs amongst Quasar binaries with respect to the relative abundance of BAL QSOs. Curiously, all of these three Quasar binaries are at similar redshifts $(z=0.834,0.858$, $z=0.892,0.894$, and $z=0.885,0.893$ for LBQS 0103-2753, SDSS 0300+0048 and PKS 0537-441, respectively).

The analysis of the cluster environment of PKS 0537-441 indicates that the BL Lac is located in a cluster as rich as Abell 
type $0-1$. The most comprehensive study on BL Lac cluster environments up to $z=0.65$ until now has been presented by Wurtz et al. (1997). They found that most BL Lacs reside in a relatively poor cluster environment of Abell type $<0$ with an indication of evolution (richer environments) towards higher redshifts. Similar conclusions have been drawn by Fried et al. (1993) for BL Lacs from the $1 \mathrm{Jy}$ sample at $z<0.8$. Thus our observations are in agreement with the observed trend. This is also supported by the detection of four galaxies at very similar redshifts (within $\Delta z \leq 0.002$ ) as the BL Lac. However, although the objects for the MOS-setups were selected serendipitously, an even higher number (10 galaxies, see Fig. 5) was found to be in the redshift range $z=0.9-1$. According to the magnitude-redshift relation by Eales (1985) discussed in Sect. 3.1.2 $m_{\mathrm{g}}(I)$ would only differ by $0.15 \mathrm{mag}$ between $z=0.893$ and $z=0.95$ (without $\mathrm{k}$-correction). We can not rule out that the enhanced galaxy density is due to a cluster at even higher redshift $(z \sim 0.95)$. Therefore PKS 0537-441 might either be projected onto a galaxy cluster at higher redshift or even be part of a large-scale structure with an extension towards PKS 0537-441.

\section{Summary}

We have presented a deep I-band image of PKS 0537-441 as well as multi-object spectroscopy in the field of PKS 0537-441. Our main results are:

1) We could not detect a host galaxy of PKS 0537-441 or any galaxy along the line-of-sight to the BL Lac down to a detection limit of $m_{I}=19.0$. None of the companion galaxies to PKS 0537-441 is a lensed background galaxy. Thus, we find neither evidence that the properties of PKS 0537-441 are affected by gravitational lensing nor that a potential group of galaxies surrounding PKS 0537-441 act as a lensing system itself.

2) The nearby environment of PKS $0537-441$ is very complex. The three major findings are: i) We detected extended offnuclear [O II] emission in PKS 0537-441. This is most likely due to photoionization by the active nucleus, although an attractive alternative - jet-cloud interaction by the counterjet in PKS 0537-441 - can not be ruled out. ii) One companion to PKS 0537-441 has a very broad, asymmetric absorbed Mg II line at $z=0.885$. This source is most likely a QSO, possibly a mini low-ionization BAL QSO. If this can be confirmed, PKS 0537-441 would be the first BL Lac being a member of a binary QSO. iii) We further found two companion galaxies within a few arcsec at similar redshifts as the BL Lac $(z=0.892$ and 0.895 vs. $z=0.893$ ), which may trigger the strong activity in this source. One of the companion galaxies is at the location, where the radio jet of PKS 0537-441 turns over.

3) PKS 0537-441 seems to be embedded in a galaxy cluster as rich as Abell type 0-1. This is supported by the detection of four galaxies at similar redshifts as the BL Lac $(\Delta z<0.002)$. Since we found serendipitously an even higher number of galaxies between $z=0.9-1$, the BL Lac may also be projected onto a galaxy cluster at somewhat higher redshift $(z \sim 0.95)$ or be part of a large-scale structure with an extension towards PKS 0537-441.
Clearly, the interpretation of the most interesting findings is difficult and somewhat speculative. High-resolution spectroscopy of the extended [O II] emission line region (possibly also in [O III]) in PKS 0537-441 with the VLT are required to unambiguously discriminate between the photoionization and jet-cloud interaction model (e.g. by looking for kinematic signatures of a counterjet). A deep high-resolution VLA image of PKS 0537-441 would be useful to search for a counterjet directly. High-resolution spectroscopy in the blue and moderateresolution spectroscopy in the far-red part of the optical spectrum or even in the NIR is necessary to confirm the presence of a (mini low-ionization BAL) QSO and to establish the presence of a binary QSO incorporating the BL Lac.

Acknowledgements. We thank the referee, Dr. Elena Pian, for a careful reading of the manuscript. It is a pleasure to thank I. Appenzeller for allocating us GTO-time for the spectroscopic observations at the VLT1. We thank also the ESO staff at the VLT and NTT for their excellent support during the observations, B. Ziegler for taking a short acquisition image of PKS 0537-441 at the VLT1 for the preparation of the MOS-setups and P. Cassaro for his VLA $1360 \mathrm{MHz}$ image of PKS 0537-441. We appreciate suggestions and discussions with M. Dietrich, S. Noll, S. Seitz, C. Tadhunter and C. Tapken during the various stages of this project. This work was supported by the Deutsche Forschungsgemeinschaft (SFB 375, SFB 439), the VW foundation, the German Federal Ministry of Science and Technology with ID-Nos. 05 2HD50A, 05 2GO20A and 05 2MU104 and the Academy of Finland (project 42697).

\section{References}

Axon, D. J., Unger, S. W., Pedlar, A., et al. 1989, Nature, 341, 631 Bechtold, J., Dobrzycki, A., Wilden, B., et al. 2002, ApJS, 140, 143 Begelman, M., Blandford, R., \& Rees, M. 1980, Nature, 287, 307

Bertin, E., \& Arnouts, S. 1996, A\&AS, 117, 393

Boisson, C., Cayatte, V., \& Sol, H. 1989, A\&A, 211, 275

Boroson, T. A., \& Meyers, K. A. 1992, ApJ, 397, 442

Cassaro, P., Stanghellini, C., Bondi, M., et al. 1999, A\&AS, 139, 601

Cowie, L. L., Songaila, A., Hu, E. M., et al. 1994, ApJ, 432, L83

Eales, S. A. 1985, MNRAS, 217, 179

Falomo, R., Melnick, J., \& Tanzi, E. G. 1992, A\&A, 255, L17

Falomo, R., Scarpa, R., \& Bersanelli, M. 1994, ApJS, 93, 125

Falomo, R. 1996, MNRAS, 283, 241

Falomo, R., \& Kotilainen, J. 1999, A\&A, 352, 85

Fried, J. W., Stickel, M., \& Kühr, H. 1993, A\&A, 268, 53

Fukugita, M., Shimasaku, K., \& Ichikawa, T. 1995, PASP, 945, 1995

Hall, P. B., Anderson, S. F., Strauss, M. A., et al. 2002, ApJS, 141, 267

Hartman, R. C., Bertsch, D. L., \& Bloom, S. D. 1999, ApJS, 123, 79

Heidt, J., \& Wagner, S. J. 1996, A\&A, 305, 42

Heidt, J. 1999, Host galaxies and environment of BL Lac objects, in ed. L. O. Takalo, \& A. Sillanpää, Proc. BL Lac Phenomenon, PASP, 159,367

Heidt, J., Nilsson, K., Sillanpää, A., Takalo, L. O., \& Pursimo, T. 1999, A\&A, 341, 683

Heidt, J., Jäger, K., Nilsson, K., et al. 2001a, The BL Lacertae object PKS 0537-441: a lens or being lensed?, AG Abstr. Ser., 18, 68

Heidt, J., Fried, J. W., Hopp, U., et al. 2001b, Host galaxies and cluster environment of BL Lac objects at $z>0.5$. In QSO hosts and their environments, ed. I. Marquez, J. Masegosa, A. del Olmo, et al. (Kluwer), 39 
Heidt, J., Jäger, K., Nilsson, K., et al. 2003a, The BL Lacertae object PKS 0537-441: a lens or being lensed? In High energy Blazar astronomy, ed. L. Takalo, \& A. Sillanpää, PASP Conf. Ser., in press

Heidt, J., Appenzeller, I., Gabasch, A., et al. 2003b, A\&A, 398, 49

Hill, G. J., \& Lilly, S. 1991, ApJ, 367, 1

Junkkarinen, V., Shields, G. A., Beaver, E. A., et al. 2001, ApJ, 549, L155

Kochanek, C. S., Falco, E. E., \& Muñoz, J. A. 1999, ApJ, 510, 590

Kotilainen, J., Falomo, R., \& Scarpa, R. 1998, A\&A, 336, 479

Lewis, G. F., \& Ibata, R. A. 2000, ApJ, 528, 650

Landolt, A. U. 1992, AJ, 104, 340

Nilsson, K., Heidt, J., Pursimo, T., et al. 1997, ApJ, 484, L107

Nilsson, K., Pursimo, T., Takalo, L. O., et al. 1999, PASP, 111, 1223

Nilsson, K., Pursimo, T., Heidt, J., et al. 2003, A\&A, 400, 95

Mortlock, D. J., Webster, R. L., \& Francis, P. J. 1999, MNRAS, 309, 836

Ostriker, J. P., \& Vietri, M. 1985, Nature, 318, 446

Oke, J. B. 1990, AJ, 99, 1621

Patnaik, A. R., Browne, I. W. A., King, L. J., et al. 1993, MNRAS, 261,435
Pian, E., Falomo, R., Hartman, R. C., et al. 2002, A\&A, 392, 407

Rector, T. A., \& Stocke, J. T. 2001, AJ, 122, 565

Romero, G. E., Surpi, G., \& Vucetich, H. 1995, A\&A, 301, 641

Romero, G. E., Cellone, S. A., \& Combi, J. A. 2000, AJ, 120, 1192

Scarpa, R., Urry, C. M., Falomo, R., et al. 1999, ApJ, 521, 134

Scarpa, R., Urry, C. M., \& Falomo, R. 2000, ApJ, 532, 740

Solórzano-Iñarrea, C., Tadhunter, C. N., \& Axon, D. J. 2001, MNRAS, 323, 965

Stickel, M., Fried, J. W., \& Kühr, H. 1988, A\&A, 206, L30

Stickel, M., Fried, J. W., \& Kühr, H. 1993, A\&AS, 98, 393

Tadhunter, C. N. 2002, Rev. Mex. Astron. Astrofis., 13, 213

Urry, C. M., \& Padovani, P. 1995, PASP, 107, 803

Vanden Berk, D. E., Richards, G. T., Bauer, A., et al. 2001, AJ, 122, 549

Veilleux, S., \& Osterbrock, D. E. 1987, ApJS, 63, 295

Wold, M., Lacy, M., Lilje, Per B., et al. 2000, MNRAS, 316, 267

Wurtz, R., Stocke, J. T., \& Yee, H. K. C. 1996, ApJS, 103, 109

Wurtz, R., Stocke, J. T., Ellingson, E., \& Yee, H. K. C. 1997, ApJ, 480,547

Wagner, S. J., \& Witzel, A. 1995, ARA\&A, 33, 163

Wrobel, J. M., \& Lind, K. R. 1990, ApJ, 348, 135 